\title{
Cryopreservation of Pacific oyster (Crassostrea gigas) larvae: Revisiting the practical limitations and scaling up the procedure for application to hatchery
}

\author{
Labbé Catherine ${ }^{1,{ }^{*}}$ Haffray Pierrick ${ }^{2}$, Mingant Christian ${ }^{3}$, Quittet Benjamin ${ }^{2}$, Diss Blandine ${ }^{4}$, \\ Tervit H. Robin ${ }^{5}$, Adams Serean L. ${ }^{6}$, Rimond Flore ${ }^{3}$, Suquet Marc ${ }^{3, *}$
}

${ }^{1}$ INRA, UR1037 LPGP, Rennes, France

2 SYSAAF, LPGP, Rennes, France

3 Ifremer, UMR 6539, PFOM Department, Station Expérimentale d'Argenton, Argenton, France

${ }^{4}$ Satmar, Barfleur, France

${ }^{5}$ Ag Research, Private bag 3123, Hamilton, New Zealand

${ }^{6}$ Cawthron Institute, Hamilton, New Zealand

* Corresponding authors : Catherine Labbé, email address : catherine.labbe@inra.fr ; Marc Suquet, email address : marc.suquet@ifremer.fr

\begin{abstract}
:
Pacific oyster Crassostrea gigas is one major species for aquaculture, and the development of breeding programs and the need for preservation of wild stock genetic resources prompted the need for larvae cryopreservation. The objective of the present study was to choose the most reliable protocol from several existing publications, to test its biological and practical limitations, and to adapt it to hatchery conditions. The selected protocol was characterized by a very slow freezing rate without seeding, and by the use of ethylene glycol and sucrose as cryoprotectant. The best survivals after thawing and rearing up to $48 \mathrm{~h}$ post fertilization (hpf) were obtained with larvae that were frozen at late trochophore (20 hpf) and early-D (24 hpf) stages. Increasing the larvae concentration in the straws and using high throughput straw filling and freezing devices did not alter the cryopreservation outcome. The whole procedure was applied to cryopreservation in a commercial hatchery (Satmar, France), and the thawed larvae yielded $9.4 \pm 4.5 \%$ survivals at 12 days post fertilization. The overall success was dampened by some variability in the larvae survival that is likely due to the physiological status of the larvae. In all, the proposed procedure is robust and reliable and can be used for cryobanking of oyster genetic resources.
\end{abstract}




\section{Highlights}

- A cryopreservation method for Pacific oyster larvae was adapted and scaled up to suit its transfer to hatchery The survival rates assessed several days after thawing display better the cryopreservation outcome than earlier stages. Cryopreservation of late trochophore to early- $D$ stages allows that enough live and functional larvae are recovered for larvae production or strain restoration.

Keywords : Oyster, Cryobanking, D stage, Ethylene glycol, High throughput, Hatchery 


\section{Introduction}

The rationale of larvae cryopreservation differs from that of gametes as the genetic resource borne by the larvae is settled at the time of the fertilization, ahead of the cryopreservation and thawing. Thus, although larvae cannot be immediately used to create new genetic variation, as would fertilization with gametes from different genetic background, larvae cryopreservation preserves the whole diploid genomes already created, thereby contributing to the maintenance of the genetic diversity for domestic and wild stocks. Since the pioneering work of Renard (1991), about 40 studies dealing with mollusk embryos or larvae cryopreservation have been published (reviewed by Martinez-Paramo et al., 2017), and the promising development rates described in several studies offer a striking contrast with fish embryo or larvae which have not been reliably cryopreserved so far. Embryo or larva cryopreservation studies in mollusk have been mainly focused on Pacific oyster. Indeed, Pacific oyster is one major species for aquaculture, with a worldwide production close to 625000 tones in 2014 (FAO, 2016). The development of embryo and larvae cryopreservation in this species would be a valuable tool for Pacific oyster farming, especially for the preservation of selective breeding genetic resources and that of special lines such as tetraploids. Pacific oyster embryo and larvae cryopreservation should also allow that hatcheries can extend their running period after the reproduction period, when gametes are no longer available although food and water temperature are still favorable to larvae production.

As for any species and cell types, cryopreservation requires the selection of optimal parameters, but the specific one to be addressed with embryos and larvae is the development stage. However, because of the difficulty to set up stage experiments, comparisons of the cryopreservation success at different stages are scarce. Pearl oyster (Pinctada fucata martensii) larvae cryopreserved at late-D stage ( $72 \mathrm{~h}$ post-fertilization) showed the best post- 
thawing survival when compared to earlier and later stages (Choi and Chang, 2003). In Pacific oyster, early embryonic stages (up to gastrula) were shown to be more sensitive to cryopreservation than early trochophore larvae (Gwo, 1995, Usuki et al., 2002). Early trochophore stage was shown to bear a better cryopreservation ability than the later D stages (Usuki et al. 2002). However, this finding is belied by the more recent work of Choi and Chang (2014) where trochophore was the worst and late-D the best stage. Apart from these scarce comparative studies, most other authors chose to study one single stage, trochophore and D stages being the most represented ones.

The other cryopreservation parameters studied include extenders, cryoprotectant types and concentrations, freezing rate, larvae concentration in straws, and thawing conditions. If seawater is most often used as an extender prior to cryopreservation, dilution of seawater with distilled water (1:4) as an extender improved the survival rate of Pacific oyster larvae after thawing (Usuki et al., 2002). Ethylene glycol as a cryoprotectant improved the survival of Pacific oyster larvae after thawing when compared to dimethyl sulfoxide (DMSO) (Choi and Chang, 2014), while Gwo (1995) gave propylene glycol as the best one. At embryonic stages, DMSO was better than glycerol, propylene glycol and ethylene glycol (Lin et al., 1999). Adding sugars (fructose, glucose, sucrose or trehalose) improved the post-thaw survival of Pacific oyster embryos and larvae by reducing the toxicity of cryoprotectants and decreasing injuries after thawing (Renard, 1991, Choi and Chang, 2014), although adding sugars gave neutral results in the study of Paredes et al. (2013). The cooling rates that were selected as the best ones differ between studies, ranging from $-0.3{ }^{\circ} \mathrm{C} / \mathrm{min}$ to $-4{ }^{\circ} \mathrm{C} / \mathrm{min}$, with or without a seeding step, and the best plunging temperature into $\mathrm{LN}_{2}$ ranged from $-30{ }^{\circ} \mathrm{C}$ to $-40{ }^{\circ} \mathrm{C}$ (Chao et al., 1997; Lin et al., 1999; Usuki et al., 2002; Paredes et al., 2013; Choi and Chang, 2014). Apparent discrepancies among authors about the best protocol within a given species are 
likely due to interactions among the tested parameters. For instance, the best survival of Pacific oyster larvae after thawing was obtained with a cooling rate of $-2{ }^{\circ} \mathrm{C} / \mathrm{min}$ with DMSO, but with a cooling rate of $-4{ }^{\circ} \mathrm{C} / \mathrm{min}$ with glycerol (Lin et al., 1999). Those divergences among findings could be created by stage-related sensitivity to different cryoprotectant and freezing conditions, together with the fact that the reported survivals were assessed from few minutes to several days after thawing.

As summarized above, several protocols with various freezing conditions for embryo and larvae cryopreservation in Pacific oyster were proposed. However, their respective efficiency measured as larvae survival after thawing was never compared using experimental conditions that were similar, including the same rearing temperature, water quality, and the same oyster strain. The objective of the present work was to compare the effectiveness of cryopreservation protocols that were already published, in order to select the most adapted one which would allow the highest survival of larvae post thawing. The robustness of the selected protocol was then assessed by studying the effect of different handling parameters which would be more suitable in hatchery conditions. This included oyster development stage, larvae concentration in straws and freezing devices. Last, the method was applied in hatchery conditions. The general strategy of this work is summarized in figure 1.

\section{Methods}

\subsection{Collection, cryopreservation and quality assessment of oyster larvae}

During the natural spawning period, Pacific oyster larvae were obtained after artificial fertilization and incubation at $19{ }^{\circ} \mathrm{C}$ into $5 \mathrm{~L}$ beakers according to Suquet et al. (2014). At trochophore stage 18 hours post fertilization (hpf), and at D stage (24 to $48 \mathrm{hpf}$ ), larvae obtained from 2 to 5 different parents and yielding development rates above $50 \%$ were 
pooled (same number of larvae from each parents). The pool was then concentrated by sieving through $20 \mu \mathrm{m}$ mesh for trochophore stage and $40 \mu \mathrm{m}$ mesh for D stages. The concentrated larvae were immediately stored on ice $\left(4^{\circ} \mathrm{C}\right)$. This cooling step did not induce any alteration of the subsequent developmental ability of the fresh trochophore larvae (data not shown). From each pool tested for cryopreservation, a fraction of the larvae was spared and immediately incubated in filtered sea water (SW) (30 to 50 larvae/mL in triplicates) to assess the fresh control survivals rates (quality of the fresh larvae). The fresh control larvae were reared in the same condition as the experimental ones (see in each section).

Except when stated otherwise, the pool of larvae was cryopreserved according to the final procedure set up in this work: One volume of larvae at 60000 larvae/mL in sea water was mixed with 1 volume cryoprotectant (20\% v/v ethylene glycol; $2 \%$ w/v polyvinylpyrrolidone 40000 average MW -PVP-40-; $400 \mathrm{mM}$ sucrose; in Milli-Q water) at $4{ }^{\circ} \mathrm{C}$. Larvae were transferred into $0.5 \mathrm{~mL}$ straws (IMV Technologies, France), and kept on ice at $4{ }^{\circ} \mathrm{C}$. The straws were cryopreserved in a programmable freezer Micro-Digitcool (IMV Technologies, France), using the following cooling profile: $-2.5^{\circ} \mathrm{C} / \mathrm{min}$ from 0 to $-10{ }^{\circ} \mathrm{C}$, hold for $5 \mathrm{~min}$ at $10{ }^{\circ} \mathrm{C},-0.3{ }^{\circ} \mathrm{C} / \mathrm{min}$ from $-10{ }^{\circ} \mathrm{C}$ to $-20{ }^{\circ} \mathrm{C},-2.5{ }^{\circ} \mathrm{C} / \mathrm{min}$ down to $-35^{\circ} \mathrm{C}$, and finally plunging into liquid nitrogen $\left(\mathrm{LN}_{2}\right)$. For thawing, the straws were plunged into a water bath $\left(37^{\circ} \mathrm{C}, 10\right.$ s). Contents of the straws were diluted in a large volume of filtered sea water $\left(2 \mathrm{~L}, 19^{\circ} \mathrm{C}\right)$ for at least 5 min to allow equilibration, and washed on $20 \mu \mathrm{m}$ mesh (trochophore stage) or 40 $\mu \mathrm{m}$ mesh (D stage). Stepwise cryoprotectant dilution into sea water did not improve the trochophore larvae survival assessed one hour post-thawing (data not shown). Larvae concentration was adjusted to 30 to 50 larvae/mL and larvae were further reared for 1 to 10 days in triplicates in $5 \mathrm{~L}$ flow-through incubator system in filtered sea water with continuous 
feeding according to Rico-Villa et al. (2008). The number of pools per experiment (biological replicates) is indicated in each section.

The cryopreservation success was estimated from motility rate, recovery rate and survival rate. The motility of the larvae was assessed $1 \mathrm{~h}$ after thawing by microscopic observation of at least 100 larvae per scoring, in triplicate. It should be pointed out that the motility parameter is arduous to assess in D larvae, as they do not display continuous and active swimming, contrarily to trochophore larvae. The motility rate (\%) of the larvae was calculated as the number of motile larvae / total number of larvae $\mathrm{x} 100$. The recovery rate (\%) was also assessed $1 \mathrm{~h}$ after thawing. It was calculated as the number of thawed larvae with a normal morphology / total number of cryopreserved larvae $\mathrm{x} 100$. This parameter encompasses the losses due to some larvae bursting during the cryopreservation process, and those due to drastic alteration in their morphology. Only the larvae displaying an almost normal size and morphology (trochophore stage) or filling the shell by more than $70 \%$ of the shell area (D stage) were scored as normal morphology. Survival rate $(\%)$ of the larvae was calculated at different stages after thawing as the number of larvae with a normal morphology / total number of surviving larvae at the beginning of the incubation x 100. Because oyster larvae had to be fed after the late-D stage (48 hpf) and that it required an algae production infrastructure, survivals of most of the cryopreservation condition were assessed at late-D stage (one day after thawing). However, in the final steps of this work, survival rates at later stages were included.

\subsection{Comparison of three existing cryopreservation protocols}

As outlined in figure 1, the three tested protocols were taken from Tervit et al. (2005) (protocol A), from Gwo, 1995 (protocol B) and from Usuki et al. (2002) (protocol C). 
Protocol A was in fact the adaptation of a protocol set up for oocyte (Tervit et al., 2005), and it was routinely used by Tervit and Adams for trochophore larvae (personal communication). The three protocols were specific with regards to their cryoprotectant composition, their freezing rate, and the seeding step. In protocol A, the cryoprotectant composition (before dilution with the larvae) was $20 \%$ (v/v) ethylene glycol, $2 \%$ (w/v) PVP-40, $400 \mathrm{mM}$ trehalose, in distilled water. The freezing rate was $-1{ }^{\circ} \mathrm{C} / \mathrm{min}$ down to $-10{ }^{\circ} \mathrm{C}$, hold for 5 min, then $-0.3{ }^{\circ} \mathrm{C} / \mathrm{min}$ down to $-35{ }^{\circ} \mathrm{C}$ prior to plunging into $\mathrm{LN}_{2}$. In protocol $\mathrm{B}$, the cryoprotectant composition was $20 \%(\mathrm{v} / \mathrm{v})$ propylene glycol in artificial sea water $(25 \mathrm{ppt})$. The freezing rate was $-2.5{ }^{\circ} \mathrm{C} / \mathrm{min}$ down to $-7{ }^{\circ} \mathrm{C}$, seeding and hold for $10 \mathrm{~s}$, then $-2.5{ }^{\circ} \mathrm{C} / \mathrm{min}$ down to $-30{ }^{\circ} \mathrm{C}$ prior to plunging into liquid nitrogen. In protocol C (Usuki et al., 2002), the cryoprotectant composition was $3 \mathrm{M}$ DMSO, $500 \mathrm{mM}$ trehalose in sea water (35 ppt). The freezing rate was $1{ }^{\circ} \mathrm{C} / \mathrm{min}$ down to $-8^{\circ} \mathrm{C}$, seeding and hold for $15 \mathrm{~min}$, then $-1{ }^{\circ} \mathrm{C} / \mathrm{min}$ down to $-35^{\circ} \mathrm{C}$ prior to plunging into $\mathrm{LN}_{2}$. All these authors chose the trochophore stage as the best one, and this is the stage that was used in this experiment. The three protocols were normalized for the larvae to cryoprotectant ratio $(1 \mathrm{v} / 1 \mathrm{v})$, the larvae concentration in cryoprotectant $(3000$ larvae/mL cryoprotectant), the $500 \mu \mathrm{L}$ straws, and for the starting temperature at $4{ }^{\circ} \mathrm{C}$. Late trochophore larvae were pooled as described (2.1.), and six independent pools were assessed. The Kryo 360-3.3 (Planer, UK) was used for programmable freezing. The recovery and motility rates were assessed $1 \mathrm{~h}$ after thawing, to allow a short recovery of the larvae prior to analysis.

\subsection{Effect of the oyster developmental stage}

Larvae were obtained as described in 2.1 and cryopreserved according to protocol A at different developmental stages post fertilization: $11 \mathrm{~h}$ (early trochophore), 18-20 h (late trochophore), $24 \mathrm{~h}$ (early-D), and $30 \mathrm{~h}$ (middle-D) (figure 1). The number of pools for the 
different stages was respectively 5, 6, 7, and 4. After thawing, larvae were incubated in $2 \mathrm{~L}$ static sea water at $19^{\circ} \mathrm{C}$ up to the late-D stage at a concentration of 30 larvae/mL.

\subsection{Effect of early-D larvae concentration in the cryoprotectant}

Larvae at early-D stage were concentrated in sea water at densities ranging from 32000 larvae/mL to 140000 larvae/mL. Within one hour after larvae concentration, samples were frozen according to the protocol A, ending up with 16000 to 70000 larvae $/ \mathrm{mL}$ cryoprotectant. After thawing, larvae were incubated in $2 \mathrm{~L}$ static sea water at $19{ }^{\circ} \mathrm{C}$ up to the late-D stage, at a concentration of 30 larvae/mL.

\subsection{Cryopreservation Scaling up}

2.5.1. Effect of the straw filling procedure and the freezing device.

The hand straw filling and the automatic filling with the MRS1 machine (IMV Technologies, France) were compared. In the hand method, straws were filled by plunging the tip of the straw into the cold larvae suspension and gentle aspiration with a vacuum pump. The MRS1 is an automatic straw-filling and ultrasound straw-sealing machine with a processing capacity of 3600 straws/hours. The larvae in cryoprotectant were transferred in the disposable plastic cone of the non refrigerated chamber of the MRS1 and processed through the tubing under vacuum according to the manufacturer's instructions. The experiment was carried out on 3 pools of early-D larvae. Thirty eight straws were filled either by hand or with the MRS1. After cryopreservation according to protocol A and thawing, larvae were reared in 2 L static sea water at $19^{\circ} \mathrm{C}$ up to the late-D stage, at a concentration of 30 larvae/mL.

The performances of two programmable freezers Kryo 360-3.3 (Planer, UK, French retailer IMV Technologies) and Micro-Digitcool (IMV Technologies, France) were compared in our 
conditions with protocol A. The Kryo 360-3.3 is a convenient programmable freezer in laboratory conditions, but it allows the cryopreservation of a limited number of straws (60 straws). On the contrary, The Micro-Digitcool (IMV Technologies, France) has a capacity of 240 straws per run. The experiment was carried out on 4 pools of early-D larvae. For each programmable freezer, 38 to 44 straws were filled. After thawing, larvae were reared in $2 \mathrm{~L}$ static sea water at $19^{\circ} \mathrm{C}$ up to the late-D stage, at a concentration of 30 larvae/mL.

\subsubsection{Effect of changing the sugar and the freezing duration}

Because trehalose is an expensive sugar that some hatcheries may want to exclude for cost reasons, replacement of trehalose by sucrose in the extender was tested. The early-D larvae were processed according to protocol A except that the cryoprotectant with $400 \mathrm{mM}$ trehalose was compared to one with $400 \mathrm{mM}$ sucrose. Speeding up the freezing step was also tested on early-D larvae. The freezing program in Protocol A (Slow) with sucrose in the extender was compared to a faster one where the step at $-1{ }^{\circ} \mathrm{C} / \mathrm{min}$ before crystallization and the step at $0.3^{\circ} \mathrm{C} / \mathrm{min}$ after the completion of crystallization were hastened (Fast) : $-2.5^{\circ} \mathrm{C} / \mathrm{min}$ from 0 to $-10{ }^{\circ} \mathrm{C}$ :, hold for $5 \mathrm{~min}$ at $-10{ }^{\circ} \mathrm{C},-0.3{ }^{\circ} \mathrm{C} / \mathrm{min}$ from $-10{ }^{\circ} \mathrm{C}$ to $-20{ }^{\circ} \mathrm{C},-2.5{ }^{\circ} \mathrm{C} / \mathrm{min}$ down to -35 ${ }^{\circ} \mathrm{C}$, and finally plunging into liquid nitrogen $\left(\mathrm{LN}_{2}\right)$. In both sets of experiments, thawed larvae were reared for up to 10 days as described in 2.1 .

\subsubsection{Transfer to hatchery}

Because the MRS1 filling and sealing machine and the Digitcool device were suitable for larvae processing, it was used for a high scale experiment carried out in a commercial hatchery (Satmar, Gatteville, France). Larvae were produced in the hatchery at $24{ }^{\circ} \mathrm{C}$. At the early-D stage, larvae were filtered and concentrated at 93000 larvae/mL prior to addition of one volume cryoprotectant (protocol A). The use of the MRS1 allowed the handling and 
filling of 426 straws in less than $20 \mathrm{~min}$. Because of the high amount of larvae to be processed, the exposure time to the cryoprotectant prior to freezing was $90 \mathrm{~min}$ at $4{ }^{\circ} \mathrm{C}$ (on ice). Close to ten million larvae were cryopreserved according to protocol $\mathrm{A}$ in one single run using the large scale freezer Digitcool (150 L) operated on the same principle as the MicroDigitcool. After thawing, larvae were incubated into $1 \mathrm{~m}^{3}$ tanks (4.12 x $10^{6}$ larvae per tank), in duplicate and reared according to the hatchery practice with micro-algae feeding. Larvae survival was counted at 5 and 12 days post fertilization (dpf).

\subsection{Statistics}

All results were expressed as mean $\% \pm$ standard deviation. When data were paired (a given larvae pool was split to test different conditions), means were compared using the non parametric Wilcoxon signed-rank test. Otherwise, means were compared using the non parametric Kruskal-Wallis test followed, when significant, by a Mann and Whitney test for comparison 2 by 2 . Significance was accepted for $\mathrm{P}<0.05$.

\section{Results}

\subsection{Comparison of three existing cryopreservation protocols}

Because the freezing rates were different among the 3 tested protocols, the cryopreservation procedure could not be performed at the same time. Therefore, in a preliminary experiment, we tested whether late trochophore larvae $(20 \mathrm{hpf})$ could be stored for at least $5 \mathrm{~h}$ at $4{ }^{\circ} \mathrm{C}$ at a density of 3000 larvae $/ \mathrm{mL}$ in sea water, in order to prevent further development of the waiting trochophore larvae. After storage, the larvae were diluted and allowed to resume development at $19{ }^{\circ} \mathrm{C}$ up to the late-D stage. The survival rates of the $\mathrm{D}$ larvae originating from cold exposed trochophore were the same as those of the $20{ }^{\circ} \mathrm{C}$ controls: $64.8 \% \pm 8.5$ and $71.0 \pm 10.5$ respectively $(n=3$ independent experiments, $\mathrm{p}>0.05$ ). 
When comparing the three cryopreservation protocols, the recovery rates were always high and not significantly different among protocols (figure 2), suggesting that whatever the freeze-thawing protocol, trochophore larvae did not burst and did not disappear upon freezing or thawing. Nevertheless, some larvae functionality was altered as motility rates were lower in the cryopreserved samples than in the fresh controls (controls $>95 \%, \mathrm{p}<0.05$ ). Motility in protocol $\mathrm{C}$ was the most variable among pools. On the contrary, protocol A gave motility rates almost twice higher than the 2 others (figure 2). Protocol A was selected for further investigation.

\subsection{Effect of oyster developmental stages on the cryopreservation outcome}

We investigated the robustness of the protocol A with regards to different larval stages. The early trochophore stage (11 hpf) yielded the lowest motilities and recovery just after thawing by comparison to the other development stages (figure 3). Although the immediate motilities and recovery rates were high from the late trochophore stage on, the movement was always slower in the thawed larvae than in the fresh controls, for the four development stages (not shown). The damages induced by the freeze-thawing stress were even more visible after the thawed larvae were incubated up to the late-D stage. Of all the thawed larvae incubated in sea water up to the late-D stage, less than one third survived (figure 3). The least resistant were the middle-D larvae cryopreserved at $30 \mathrm{hpf}$, as their survival rate was significantly lower than that of any other cryopreservation stage. The survival rates of the fresh controls exposed to the cryoprotectant ranged from 61 to $85 \%$ at late-D stage $(78.0 \% \pm 5.0)$. Whatever the cryopreservation stage, all cryopreserved larvae had a survival rate lower than that of the fresh controls. The early-D larvae stage (24 hpf) was chosen for the next experiments. Indeed, in the prospect of application to hatchery conditions, the 24 hpf stage is convenient because it 
corresponds to the first tank draining and sorting of the live versus developmentally arrested larvae, and this stage was among the two stages yielding the best performances after cryopreservation.

\subsection{Effect of the D larvae concentration in the cryoprotectant}

Cryopreservation of early-D larvae according to protocol A was tested at one low larvae concentration (Low: 33000 larvae/ml cryoprotectant) and at one high larvae concentration (High: 64000 larvae/mL cryoprotectant) (n=3 pools). We observed that the recovery rates were lower in the Low concentration groups than in the High concentration groups: $65.3 \pm 2.1$ and $80.7 \pm 8.1$ respectively $(\mathrm{p}<0.05)$, but the survival rates at late-D stage were not different between Low and High groups: $70.0 \pm 10.6$ and $68.3 \pm 17.9$ respectively $(\mathrm{p}>0.05)$. To confirm this result, the data collected over several months of independent experiments were processed in order to assess whether larvae concentration and survival are correlated. Within the concentration range tested (16000 to 70000 larvae/mL cryoprotectant), no significant alteration was induced by the highest larvae concentrations or by the lowest ones. Indeed, no correlation between the recovery or survival and the larvae concentration was observed (Figure 4). This means that within the tested concentration range, there was no toxicity related to overcrowding the straws. This result confirms that increasing the larvae concentration prior to cryopreservation was not deleterious in our conditions.

\subsection{Effect of the straw filling process and the freezing device.}

We observed that the two straw filling processes did not yield different cryopreservation outcome. The survival rates at late-D stage were $54.0 \pm 14.4$ for the hand filling process and $65.0 \pm 3.1$ for the MRS1 filling process $(n=3$ pools, $p>0.05)$. Therefore, the extra manipulation at room temperature and the exposure to the plastic equipment required with the 
MRS1 were not deleterious.

Two freezing devices with highly different straw processing capacity were compared. The cryopreservation outcome was not different whatever the freezing device used (Table 1), although in this set of experiments, the survival rates were highly variable with both the Kryo 360-3.3 (Planer, UK) and the Micro-Digitcool (IMV Technologies, France). The Digitcool device was used for further experiments. Indeed, with the Kryo 360-3.3, the straws are to be clipped one by one to the holders prior to insertion inside the cooling chamber. It is time consuming and does not allow the processing of large larvae samples.

\subsection{Adaptation to hatchery requirements}

The transfer of a cryopreservation protocol to hatchery prompted several adaptations of protocol A. Larvae cryopreservation in hatchery conditions required that the volumes of cryoprotectant be greater $(>250 \mathrm{~mL}$ ) than in laboratory conditions, and the cost of the components is at risk to become a relevant issue. Substituting the very expensive trehalose by sucrose was tested, because Choi and Chang (2014) had shown that sucrose was suitable for cryopreservation of Pacific oyster larvae. As shown in figure 5, the protocol A extender with either trehalose or sucrose yielded the same survivals after cryopreservation, whatever the rearing duration post-thawing.

The second limitation that was raised for application to hatchery is the duration of the freezing program. In protocol $\mathrm{A}$, it lasts almost $2 \mathrm{~h}$ when including the freezing duration and chamber warming up afterwards. A shorter program (Fast) which would allow that more cryopreservation sequences be run within the same working time frame was compared to the one used in protocol A (Slow). As shown in figure 6, both freezing rates yielded the same 
survivals after cryopreservation. This means that speeding up the freezing rate before extracellular ice crystallization occurs, and then once most of the whole crystallization is achieved, was not deleterious. It is likely that restricting the very slow $-0.3{ }^{\circ} \mathrm{C} / \mathrm{min}$ rate to the sensitive steps of crystallization and dehydration was enough to ensure the cryoprotection. In a separate experiment, the programmable freezer was replaced by the more easily available raft device. The straws were horizontally laid on a raft $3 \mathrm{~cm}$ above liquid nitrogen in a closed tank for $10 \mathrm{~min}$, prior to plunging into liquid nitrogen. We observed that such a fast freezing was totally deleterious in our conditions. All larvae were dead upon thawing: $0 \%$ motility upon thawing, and $0 \%$ survival at late-D stage in all the replicates.

\subsection{Transfer of the procedure to hatchery}

Although the whole procedure had been set up in a hatchery practice perspective, it had to be tested for real in a hatchery, because the volumes treated for fertilization and development are out of scale compared to the usual laboratory conditions. In hatchery, the cryopreserved larvae displayed much lower survival rates than the fresh controls when assessed 5 and $12 \mathrm{dpf}$ (Table 2). However, the final survival of the thawed larvae was still high when compared to most of the results obtained previously in our laboratory conditions (above $9 \%$ at $12 \mathrm{dpf}$, table 2). Beside, the survival of the fresh and cryopreserved larvae decreased only little between 5 and 12 dpf. This indicates that most of the damage induced by cryopreservation triggered the death of the larvae within the first 4 days after thawing.

\section{Discussion}

\subsection{Rating of the cryopreservation success}

In this work, a great number of cryopreservation conditions were screened, with many larvae replicates in each experiment, in order to ensure that the final method was robust and reliable 
in the perspective of application to hatcheries. This prompted the need for simple criteria in order to evaluate the thawed larvae quality. The efficiency of most of the tested and adapted protocols was assessed at late-D stage. This stage allowed the larvae to recover after thawing for one day in the rearing tanks, and it allowed the expression of the first alterations due to cryopreservation. Another advantage of this late-D stage is that the larvae have not reached the first feeding stage yet, and this reduces the infrastructure needed to cultivate them. It is known from earlier work (Suquet et al., 2012) that at this stage, all damage induced by cryopreservation is not revealed by the survival rates only. Indeed, although alive and displaying ciliated movement, thawed larvae have a reduced movement velocity, and the flesh in D larvae can be drastically retracted, thereby inducing a reduced area of the larvae (Suquet et al., 2012). This is why the calculation of the survival rate in our conditions excluded all the larvae which had a body area too highly reduced. In all, the survival rate at late-D stage allowed a straightforward assessment of the potential of the thawed larvae. Moreover, this simple quality assessment combined with recovery and motility at thawing allowed us to set up a procedure which yielded acceptable larvae survival in hatchery.

We confirmed previous results by Suquet et al. (2012) which showed that the survival rates of the thawed larvae is decreasing after the first days of rearing. It is likely that it is the inability of the most damaged larvae to feed which is responsible for the dramatic losses that we observed after the late-D stage. We showed previously that after the first 2 weeks, the survival and growth between fresh and cryopreserved larvae are no longer different (Suquet at al., 2014), indicating again that most damage induced by cryopreservation was expressed before. Therefore, accurate survival rate should be assessed during these first 2 weeks, preferably around 5-7 dpf. This would be the best stages to encompass most of the cryopreservationinduced alterations, although these stages require that a micro-algae production facility is 
running in order to provide for the larvae feeding.

\subsection{Choice of the most promising protocol}

Our objective was to compare three existing cryopreservation protocols for Pacific oyster larvae, in order to select the best one. The true comparison, with the same larvae sample, of protocols bearing different freezing rates was in fact difficult to achieve. Indeed, the larvae development had to be stalled during the cryopreservation with the first protocol, so that the larvae for the second and third protocol were still at the same stage as the first one. This may not be without consequences, whatever the precautions taken. Second, each published protocol was using different larvae concentration. Some mortality can be attributed only to a density effect during cryopreservation, and not to the overall efficiency of the protocol. This is why the same larvae concentration was used for all three protocols, although it induced a bias in the absolute comparison. Last, the seeding procedure and the freezing devices are different among laboratories, and it is not always possible to exactly reproduce another author's procedure. Taking into account the concerns listed above, we chose the protocol A by Tervit et al. (2005) which resulted in higher larval motilities than those by Gwo (1995) or Usuki et al. (2002). In their own work, Gwo (1995) reported $7 \%$ survival at the veliger stage (fresh controls $63 \%$ ). Usuki et al. (2002) reported $17.5 \%$ survival 4 days after thawing (fresh control not given). Tervit et al. (2005) obtained $18 \%$ D larvae from cryopreserved oocytes (fresh control $45 \%$ ). It is difficult to ascertain what made protocol A better than the others in our experiment, especially when they differ by so many parameters such as the permeating cryoprotectant, the sugar, the freezing rate, the crystallization trigger etc. In all, one great advantage of protocol $\mathrm{A}$ is the lack of manual seeding, allowing less variability and better potential for scaling up in hatchery conditions. 


\subsection{Importance of the larval stages for cryopreservation}

The stage at which cryopreservation is achieved may differ among hatcheries, depending on the organization of the fertilization workflow, and on the rearing temperature in the tanks. This is why we tested whether the cryopreservation could be processed on stages that are different from the most studied trochophore stage. After fertilization, bivalve mollusks undergo a brief embryonic stage going through morula, blastula and gastrula stages (from 7 to $12 \mathrm{~h}$ in Pacific oyster at $25^{\circ} \mathrm{C}$ ) after which a free swimming trochophore larva is released. After 18 to $24 \mathrm{~h}$ in Pacific oyster, a D-shaped larva with two symmetric shells is formed. This larval stage will be associated with first feeding 48 to $72 \mathrm{hpf}$. The later pedi-veliger (or umboveliger) stage will be associated with shell asymmetry when the valve's beak is formed (from 4 to 14 dpf in Pacific oyster). Metamorphosis which changes the veliger larvae into settled larvae attached to collectors (spat) will take place after 15-30 days in Pacific oyster. Among all those stages, the ones tested in our study were within the first 2 days after fertilization. This is before the larvae require micro-algae feeding, thereby allowing an easier processing of the larvae in the hatcheries prior to cryopreservation. When taking all quality parameters into account, we demonstrated that the $20 \mathrm{hpf}$ (late trochophore) and $24 \mathrm{hpf}$ (early-D larvae) stages were the best ones in Pacific oyster, compared to earlier and later developmental stages. This result is especially interesting because the transition between late trochophore and D larvae can span over few hours. As both stages are fit for cryopreservation, this gives some flexibility to the operator with regards to the cryopreservation time. All larvae can be cryopreserved even if some have not undergone shell building (D stage) yet. Although our study was restricted to early stages, later stages should not be totally overlooked. It was shown by Choi and Chang (2014) that stages up to umbo-veliger (12 dpf) still had high survival rates, at least when assessed 1 hour post-thawing. If these larvae have the ability to survive longer, this will open other possibilities for oyster farmers. Indeed, it will allow the 
cryopreservation of the spare larvae that would otherwise be discarded during the density adjustments phases.

\subsection{Lack of larvae concentration effect for cryopreservation}

The remarkable robustness of our cryopreservation protocol with regards to the D larvae concentration in the cryoprotectant (16 000 to 70000 larvae/mL) is especially interesting with regards to cryobanking costs, when the number of straws should be reduced to the lowest. To our knowledge, the highest concentration that has been tested in Pacific oyster is 28000 trochophore/mL (Usuki et al., 2002). In Crassostrea virginica, Paniaga-Chavez and Tiersch (2001) had shown that in macrotubes, the lower the trochophore larvae concentration, the better the survivals at thawing, but the ranges in the concentrations of larvae that were tested by these authors were much broader (10 to 100000 larvae/mL) than ours. Only the highest concentrations in Paniaga-Chavez and Tiersch (2001) can compare with our study, and it cannot be excluded that much lower concentrations in our conditions would have yielded the same favorable effect as in Paniaga-Chavez and Tiersch (2001). Still, we did not observe in our conditions the losses that Paniaga-Chavez and Tiersch (2001) observed between their two highest tested concentrations (10 000 and 100000 larvae/mL). We can proposes that such lack of alteration whatever the concentration was obtained because the larvae were maintained on ice once they were concentrated, and later on once they were mixed with the pre-cooled cryoprotectant. The likely decrease in larvae metabolism may have prevented any damage due to overcrowding and lack of oxygen. It should be noted however that the highest concentration are more difficult to process during the filtering and pipetting. In our conditions, we considered that a concentration of 30000 larvae/mL in cryoprotectant is a good compromise between the larvae number in the straws and the easy processing of the larvae suspension. 


\subsection{Success of the scaling-up adjustments}

In this work, all adjustments that were tested in order to adapt protocol A to hatchery conditions had no significant impact on the cryopreservation outcome, and above all, they did not induce any detectable alteration. The successful mechanization of the cryopreservation protocol which included the use of high throughput filling and freezing devices will favor its transfer to the industry. Replacement of trehalose by sucrose without loss in cryoprotection is also interesting as it will reduce the costs of the extender by more than 50 fold. Trehalose is a natural disaccharide that was originally used to protect cell membranes during dehydration (Crowe et al., 1996). It was used to provide some protection during cryopreservation of Pacific oyster trochophore larvae (Usuki et al., 2002, Paredes et al., 2013), although no clear advantage of trehalose could be found when compared to extenders without any sugars (Paredes et al., 2013). That sucrose provides the same cryoprotection as trehalose has been already observed in fish sperm (Maisse, 1994), and Choi and Chang (2014) have shown that sucrose was a valuable additive to ethylene glycol for cryopreservation of Pacific oyster larvae. It is difficult to clarify whether sucrose and trehalose interchangeability is due to their physical properties during freeze-thawing (Kent et al., 2017), or to the lack of effect of sugar addition. However, it was shown that monosaccharides such as glucose or fructose do not yield the same cryoprotection as the disaccharide sucrose (Choi and Chang, 2014). Speeding up the freezing program will also facilitate the use of the procedure in the industry. Our results showed that an ultra-fast and non-controlled freezing rate such as the one obtained with the raft method cannot be used. When considering the size and the complexity of the cryopreserved larvae, it is likely that these organisms required some extensive dehydration prior to intracellular freezing (Labbe et al., 2013), and this requirement was met in both programmable freezing rates thanks to a holding step at $-10^{\circ} \mathrm{C}$ and a very slow freezing rate 
afterwards.

\subsection{Overall success for the cryopreservation of early-D larvae}

Although the absolute survival rates obtained in the hatchery experiment is low $(<10 \%$ at 12 dpf), the number of surviving larvae was in fact very high thanks to the high amount of larvae that was cryopreserved in each straw. This survival rate will still provide an excess of live larvae if these were to be incubated in hatchery tanks for production. The survivals that were achieved in our laboratory were much lower (about $1-2 \%$ at $10 \mathrm{dpf}$ ). This is surprising when considering that the volumes produced and treated were much less demanding in laboratory conditions. It cannot be excluded that the incubation conditions were more suitable in the large hatchery tanks. Water temperature was different as well. But more likely, the cause is the variability of the living material that was used in the different experiments. On the fresh larvae, we had already observed that their ability to undergo the transition between trochophore and D was highly variable, and in the present work, we have tried to reduce this variability by excluding from the pools all spawns that had low survival rates the day after fertilization, and by mixing the offspring from of 2 to 5 different breeders. Our previous work (Suquet et al., 2012) had also shown that post-thawing survivals could vary between 2 and 23 $\%$ at $7 \mathrm{dpf}$ in independent experiments. In all, the challenge of the cryopreservation may have acted as an enhancer of the initial biological fragility of the larvae in some samples, and it may have displayed some variability that was not seen on fresh larvae.

\section{Conclusion}

In conclusion, the present work confirms that the cryopreservation of Pacific oyster larvae is biologically and technically possible. We set up a procedure which is adapted to hatchery conditions, although the survival of the thawed larvae is still low. As it is, the larvae survival 
obtained after cryopreservation should already allow the genome preservation of wild Pacific oysters and that of selected individuals from breeding programs. We believe that the best candidate for optimization of the larvae survival will be revealed by a deeper knowledge of the biological features responsible for larvae ability to sustain the freezing-thawing stress, making it a biological rather than a technological issue.

\section{Acknowledgements:}

This work was supported by CRECHE (Ofimer-FEP 136/08/C, 30906-2009), CRYOAQUA (French CCRB/IBiSA 2009-2011), and by the French PAI CRB Anim project «Investissements d'avenir», ANR-11-INBS-0003 (2012-2019). The authors acknowledge the major input of the technical team of Ifremer's facilities in Argenton, including Isabelle Quéau and Dominique Ratiskol for micro algae production. They allowed that larvae survival be assessed after the first feeding stage, thanks to excellent skills and care in larval rearing and feeding procedures. The contribution of IMV technologies in providing helpful technical support and in lending freezing equipment in the scaling up experiments was highly appreciated.

\section{Figure captions}

Figure 1. General roadmap followed in the present work.

The work was separated into three sections: the choice of the best protocol from published protocols (Tervit et al., 2005; Gwo, 1995; Usuki et al., 2002), the scaling up of the procedure using automated machines, and the adaptation and application to hatchery practice.

The vertical black line follows the condition chosen after each test.

Hpf: hour post fertilization; MRS1: automatic straw filling machine from IMV Technologies company; Micro-digitcool: programmable freezer with racks holding a 240 straws capacity; 
Kryo 360: programmable freezer with stem holders bearing a 60 straws capacity. See section

2.2. for extender composition and freezing rates in protocols A, B and C. See section 2.5.2. for the description of the SLOW and FAST freezing programs.

Figure 2. Effect of 3 cryopreservation methods on the recovery and motility rates of $20 \mathrm{hpf}$ trochophore larvae $1 \mathrm{~h}$ after thawing. A: method according to Tervit et al. (2005); B: method according to Gwo (1995); C: method according to Usuki et al. (2002). Bars represent the mean $\% \pm \mathrm{SD}, \mathrm{n}=6$. Different superscripts indicate significant differences for a given parameter. Motilities of the fresh controls were always above $95 \%$ (not shown on the figure).

Figure 3. Effect of 4 larval stages at cryopreservation on the motility and recovery rates $1 \mathrm{~h}$ after thawing, and on the survival rate after rearing up to the late-D stage (48 hpf). Cryopreservation was performed according to protocol A (Tervit et al., 2005). Early and Late trocho: trochophore larvae frozen at $11 \mathrm{hpf}$ and $20 \mathrm{hpf}$ respectively; Early-D and Middle-D: D larvae frozen at $24 \mathrm{hpf}$ and $30 \mathrm{hpf}$ respectively. Bars represent the mean $\% \pm \mathrm{SD}, \mathrm{n}=4$ to 7 depending on the stage. Different superscripts indicate significant difference between stages within for a given parameter.

Figure 4. Effect of the early-D larvae concentration in the cryoprotectant on the recovery $1 \mathrm{~h}$ after thawing and on the survival rate at late-D stage (48 hpf). Larvae were cryopreserved according to protocol A. Values are individual \% of 17 different larvae pools. No significant correlation was observed between the concentration and the quality of the thawed larvae.

Figure 5: Comparison of sucrose and trehalose in the freezing extender for cryopreservation of early-D larvae. Results are mean $\% \pm$ SD of 3 independent pools. Recovery: recovery rate 
$1 \mathrm{~h}$ after thawing; Surv.: Survival at different hours and days post fertilization (hpf, dpf).

Different letters indicate significant differences for a given developmental stage.

Figure 6: Comparison of two freezing rates for cryopreservation of early-D larvae. Results are mean $\% \pm \mathrm{SD}$ of 3 independent pools. Recovery: recovery rate $1 \mathrm{~h}$ after thawing; Surv.: Survival at different hours and days post fertilization (hpf, dpf). Different letters indicate significant differences for a given developmental stage.

\section{Tables}

Table 1. Comparison of two programmable freezing devices for the cryopreservation of earlyD larvae.

\begin{tabular}{rcc}
\hline Freezing Machine & Kryo 360-3.3 & $\begin{array}{c}\text { Micro- } \\
\text { Digitcool }\end{array}$ \\
\hline Motility at thawing (\%) & $74.0 \pm 8.3$ & $89.0 \pm 7.1$ \\
Recovery at thawing (\%) & $80.8 \pm 9.8$ & $80.3 \pm 16.7$ \\
Survival at late-D stage (48 & $65.0 \pm 28.9$ & $67.6 \pm 30.3$ \\
\hline hpf) (\%) &
\end{tabular}

Larvae were cryopreserved according to protocol A. Motility and recovery rates (\%) were assessed $1 \mathrm{~h}$ after thawing. Survival rate of the thawed larvae (\%) was assessed after rearing up to the late-D stage (48 hpf, one day after thawing). Hpf: hours post fertilization. Kryo 3603.3: programmable freezer with stem holders bearing a 60 straws capacity; Micro-Digitcool: programmable freezer with racks holding a 240 straws capacity; Values are mean $\% \pm \mathrm{SD}$, $\mathrm{n}=4$. No significant differences were observed within rows.

Table 2. Cryopreservation of early-D larvae in hatchery 


\begin{tabular}{rccccc}
\hline & $\begin{array}{l}\text { Motility at } \\
\text { thawing }(\%)\end{array}$ & $\begin{array}{l}\text { Recovery at } \\
\text { thawing }(\%)\end{array}$ & $\begin{array}{l}\text { Survival at } \\
\text { late-D stage } \\
(48 \mathrm{hpf})(\%)\end{array}$ & $\begin{array}{l}\text { Survival at 5 } \\
\text { dpf }(\%)\end{array}$ & $\begin{array}{l}\text { Survival at } \\
12 \mathrm{dpf}(\%)\end{array}$ \\
\hline Fresh larvae & NA & NA & NA & $56.9 \pm 0.9$ & $52.5 \pm 3.5$ \\
$\begin{array}{r}\text { Cryopreserved } \\
\text { larvae }\end{array}$ & $87.5 \%$ & $74.0 \%$ & $78.9 \pm 12.0$ & $11.7 \pm 2.4$ & $9.4 \pm 4.5$ \\
\hline
\end{tabular}

Larvae were cryopreserved according to protocol A in a commercial hatchery (SATMAR, France). Motility and recovery rates (\%) were assessed $1 \mathrm{~h}$ after thawing. The thawed larvae were then reared in hatchery condition. Survival rate (\%) was assessed at late-D stage (48 hpf, one day after thawing), $5 \mathrm{dpf}$ and $12 \mathrm{dpf}$ (4 and 11 days after thawing). Hpf: hours post fertilization; dpf: days post fertilization. NA: not assessed. Values are mean $\% \pm \mathrm{SD}, \mathrm{n}=2$

\section{References}

Chao N.H., Lin T.T., Chen Y.J., Hsu H.W., Liao I.C., 1997. Cryopreservation of late embryos and early larvae in the oyster and hard clam. Aquaculture 155, 31-44.

Choi, Y.H., Chang, Y.J., 2003. The influence of cooling rate, developmental stage, and the addition of sugar on the cryopreservation of larvae of the pearl oyster Pinctada fucata martensii. Cryobiology 46, 190-193.

Choi, Y.H., Chang, Y.J., 2014. Influences of developmental stages, protective additives and concentrations of cryoprotective agents on the cryopreservation of Pacific oyster (Crassostrea gigas) larvae. Cryoletters 35, 495-500.

Crowe, L.M., Reid, D.S., Crowe, J.H., 1996. Is trehalose special for preserving dry biomaterials? Biophys J 71, 2087-2093. 
FAO: Food and Agriculture Organization of the United Nations. Cultured aquatic species. $\begin{array}{lllll}\text { Information } & \text { program. } & \text { Accessed }\end{array}$ http://www.fao.org/fishery/culturedspecies/Crassostrea_gigas/en

Gwo, J., 1995. Cryopreservation of oyster (Crassostrea gigas) embryos. Theriogenology. 43,1163-1174.

Kent, B., Hunt, T., Darwish, T.A., Hauss, T., Garvey, C.J., Bryant, G., 2014. Localization of trehalose in partially hydrated DOPC bilayers: insights into cryoprotective mechanisms. J R Soc Interface 11.

Labbé, C., Robles, V., Herraez, M.P., 2013. Cryopreservation of gametes for aquaculture and alternative cell sources for genome preservation, in: G. Allan, G. Burnell (Eds.), Advances in aquaculture hatchery technology. Woodhead Publishing Limited, CambridgeLin, T.T., Chao, N.H., Tung, H.T., 1999. Factors affecting survival of cryopreserved oyster (Crassostrea gigas) embryos. Cryobiology 39, 192-196.

Martínez-Páramo, S., Horváth, Á., Labbé, C., Zhang, T., Robles, V., Herráez, P., Suquet, M., Adams, S., Viveiros, A., Tiersch, T.R., Cabrita, E., 2017. Cryobanking of aquatic species. Aquaculture 472, 156-177.

Paniagua-Chavez, C.G., Tiersch, T.R., 2001. Laboratory studies of cryopreservation of sperm and trochophore larvae of the Eastern oyster. Cryobiology 43, 211-223.

Paredes, E., Bellas, J., Adams, S.L., 2013. Comparative cryopreservation study of trochophore larvae from two species of bivalves: Pacific oyster (Crassostrea gigas) and Blue mussel (Mytilus galloproviricialis). Cryobiology 67, 274-279.

Renard, P. , 1991. Cooling and freezing tolerances in embryos of the Pacific oyster, Crassostrea gigas: methanol and sucrose effects. Aquaculture 92, 43-57. 
Rico-Villa, B., Woerther, P., Mingant, C., Lepiver, D., Pouvreau, S., Hamon, M., Robert, R., 2008. A flow-through rearing system for ecophysiological studies of Pacific oyster Crassostrea gigas larvae. Aquaculture 282, 54-60.

Suquet, M., Le Mercier, A., Rimond, F., Mingant, C., Haffray, P., Labbe, C., 2012. Setting tools for the early assessment of the quality of thawed Pacific oyster (Crassostrea gigas) D-larvae. Theriogenology 78, 462-467.

Suquet M., Rimond F., Cosson J., Wilson-Leedy J., Lebrun L., Queau I., Mingant C., Fauvel c., 2013. Effect of age and environmental conditions on the movement characteristics of Pacific oyster (Crassostrea gigas) trochophores. J. Appl. Ichthyol. 29, 1145-1148.

Suquet, M., Labbé, C., Puyo, S., Mingant, C., Quittet, B., Boulais, M., Queau, I., Ratiskol, D., Diss, B., Haffray P., 2014. Survival, growth and reproduction of cryopreserved larvae from a marine invertebrate, the Pacific oyster (Crassostrea gigas). Plos one, 9 (4).

Tervit, H.R., Adams, S.L., Roberts, R.D., McGowan, L.A., Pugh, P.A., Smith, J.F., Janke, A.R., 2005. Successful cryopreservation of Pacific oyster (Crassostrea gigas) oocytes. Cryobiology 51 142-151.

Usuki, H., Hamaguchi, M., Ishioka, H., 2002. Effects of developmental stage, seawater concentration and rearing temperature on cryopreservation of Pacific oyster Crassostrea gigas larvae. Fish Sc 68, 757-762. 
1) Choice of the best protocol from published procedures

Protocol A from

Tervit et al. (2005)

Protocol B from

Gwo (1995)
Protocol C from

Usuki et al. (2002)

2) Testing of different developmental stages for cryopreservation

Trocho early

(11 hpf)
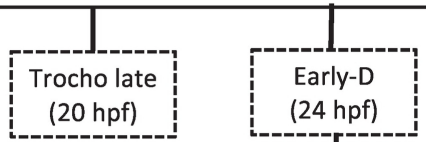

Middle-D

(30 hpf)

3) Seeking the optimal larvae concentration for cryopreservation

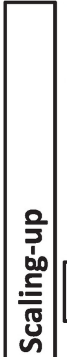

4) Testing the MRS1 automatic filling versus hand filling of the straws

5) Testing the programmable freezer type
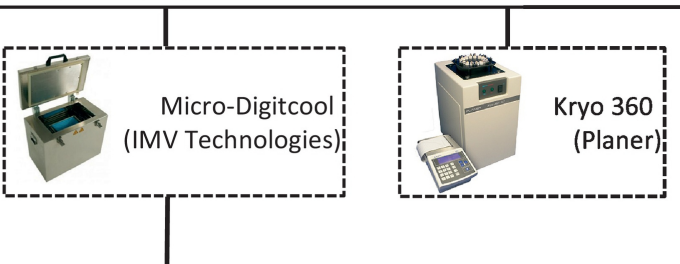

6) Application to hatchery (SATMAR, France)

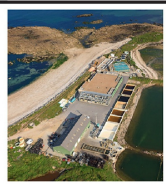

7) Testing the substitution of trehalose by sucrose in the extender
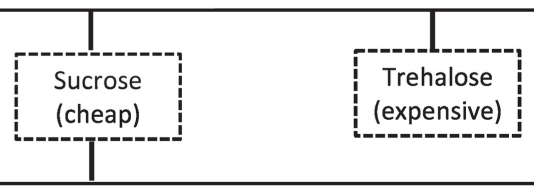

8) Testing the speeding up of the freezing step

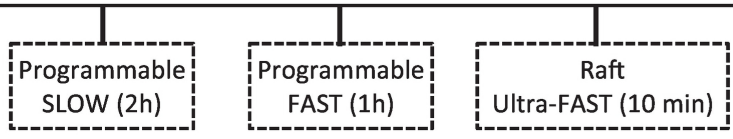




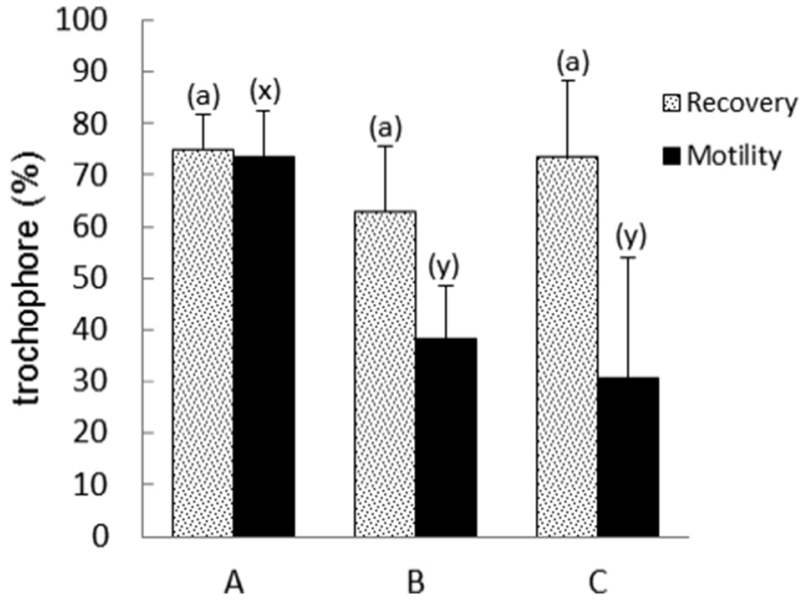

Figure 2 


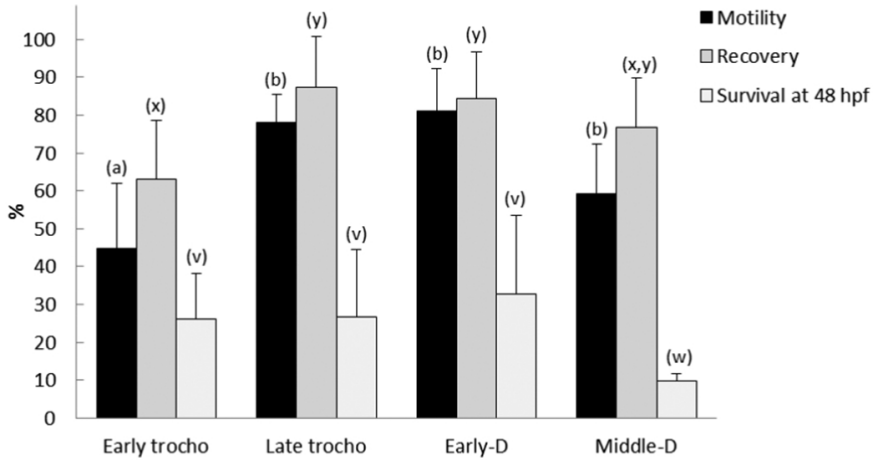

Figure 3 


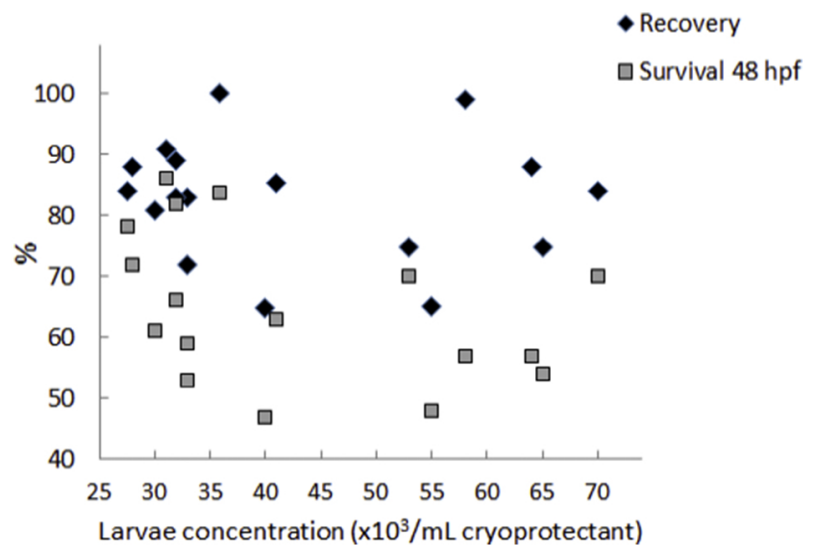

Figure 4 
- Fresh

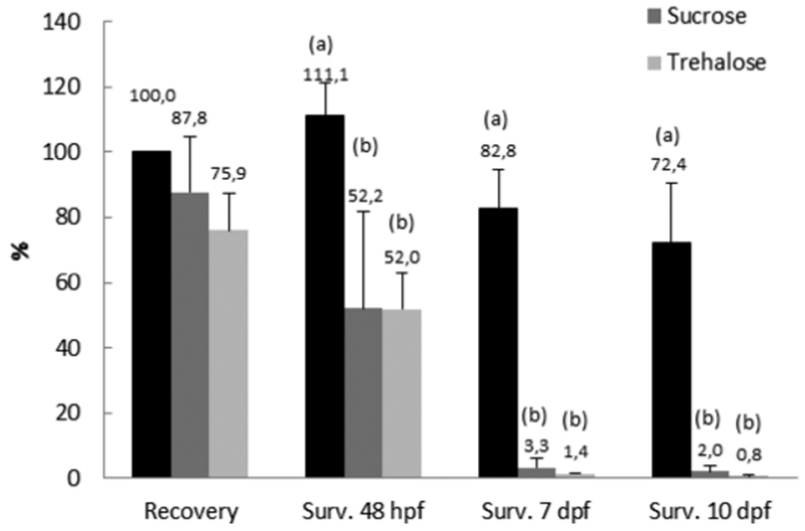

Figure 5 


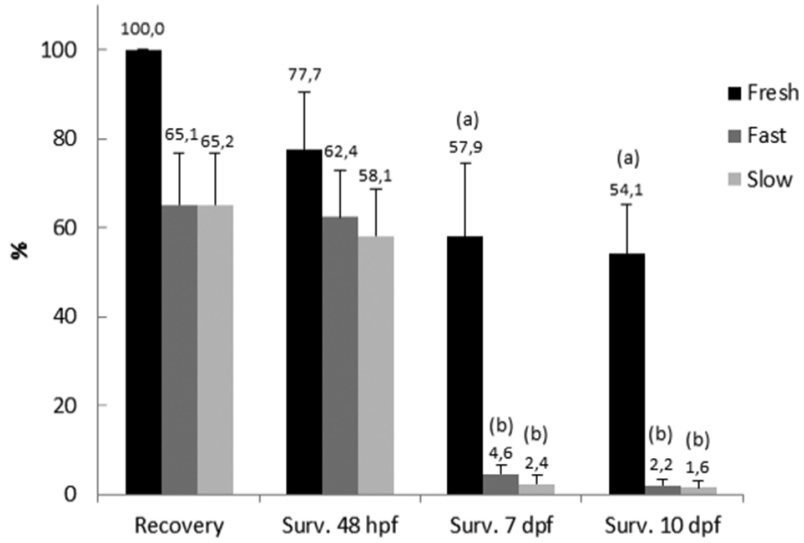

Figure 6 